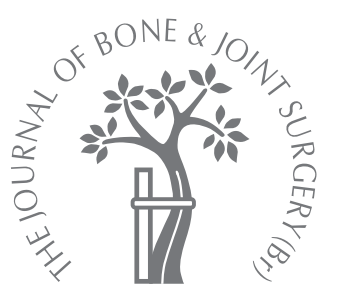

F. Middleton,

J. Coakes,

S. Umarji,

S. Palmer,

R. Venn,

S. Panayiotou

From Worthing and

Southlands NHS

Trust, Worthing,

England

F. Middleton, MBBS, FRCS(Tr \& Orth), MA(Oxon), Specialist

Registrar in Trauma and

Orthopaedics

St Peter's Hospital, Guilford

Road, Chertsey KT16 OPZ, UK.

w. Coakes, BM, MRCP,

Specialist Registrar in

Anaesthesia

Southampton General

Hospital, Tremona Road,

Southampton SO16 6YD,

United Kingdom.

In S. Umarji, BMBCh, MRCS, MA(Oxon), Specialist Registrar

in Trauma and Orthopaedics

St George's Hospital,

Blackshaw Road, Tooting

London SW17 0ST, UK.

- S. Palmer, MBBS, MSc,

FRCS(Tr \& Orth), Consultant in

Trauma and Orthopaedics

n. Venn, MD, FRCA,

Consultant in Anaesthesia \&

Intensive Care

- S. Panayiotou, MBBS, FRCA,

Consultant in Anaesthesia

Worthing and Southlands NHS

Trust, Lyndhurst Road,

Worthing, West Sussex, BN11

2DH, UK.

Correspondence should be sent to Miss F. Middleton at 18

Wodeland Avenue, Guildford

GU2 4JX, UK; e-mail:

fionamiddleton@doctors.org.

uk

(C)2006 British Editorial Society of Bone and Joint Surgery doi:10.1302/0301-620X.88B12. $17740 \$ 2.00$

$J$ Bone Joint Surg [Br]

2006;88-B:1603-5.

Received 7 February 2006;

Accepted after revision 16

August 2006

\title{
The efficacy of intra-articular bupivacaine for relief of pain following arthroscopy of the ankle
}

\begin{abstract}
The intra-articular injection of local anaesthetic is frequently used for pain relief after arthroscopy. There is, however, no published evidence of the analgesic effect of bupivacaine in the ankle. In a randomised, double-blind study, 35 patients undergoing arthroscopy of the ankle were allocated to receive intra-articular saline or bupivacaine. Pain was assessed using pain scores and additional analgesic requirements. Intra-articular bupivacaine had a significant analgesic effect in the immediate post-operative period, reducing pain scores and the need for additional analgesics.
\end{abstract}

We recommend the use of intra-articular bupivacaine for post-operative analgesia in ankle surgery.

Arthroscopy of the ankle is performed routinely for diagnostic and therapeutic purposes. ${ }^{1}$ The procedure is commonly carried out as a day case and effective analgesia is essential both immediately after operation and following discharge from hospital. ${ }^{2}$

Local anaesthetic is frequently injected intraarticularly and around the sites of the portals at the end of arthroscopic procedures to provide analgesia. Studies of intra-articular analgesia following arthroscopy of the knee have demonstrated that bupivacaine is both safe and effective $^{3-5}$ Other drugs have been used via this route, including opioids, but the results have been equivocal. ${ }^{6,7}$

Only one previous study has assessed the use of intra-articular drugs for arthroscopy of the ankle. Rasmussen and $\mathrm{Kehlet}^{8}$ compared the use of a combination of bupivacaine, morphine and methylprednisolone with a placebo (saline) and found a reduction in pain and impairment of function. However, this combination has raised concerns with regard to infection owing to the inclusion of steroids, with this study describing one case of septic arthritis in the group. ${ }^{8}$ It is not possible to determine which of the drugs used in the combination provided effective analgesia.

Although studies of post-operative analgesia in arthroscopy of the knee have demonstrated that intra-articular bupivacaine is safe and effective, this has not been proven for arthroscopy of the ankle. ${ }^{3}$

\section{Patients and Methods}

We carried out a prospective, randomised, double-blind clinical trial at a single centre to assess the analgesic effect of intra-articular bupivacaine on post-operative pain following arthroscopy of the ankle as a day case. Each patient undergoing the procedure was randomly assigned to receive either intra-articular bupivacaine or normal saline following completion of the operation. There were 17 patients in the bupivacaine group, and 18 in the saline group. Both the patient and the evaluator remained blinded as to the group assignment. The study protocol was reviewed and approved by the local research ethics committee and all patients gave informed consent.

Patients over the age of 18 years were eligible for inclusion. Exclusion criteria included allergies to any of the drugs used, the use of oral opioids or non-steroidal anti-inflammatory drugs (NSAIDS) in the previous seven days, spinal or epidural anaesthesia/analgesia, and pregnancy. The circulating theatre nurse carried out the randomisation during the operation using a sealed envelope technique.

All patients received a premedication dose of dispersible diclofenac $100 \mathrm{mg}$ and a standardised general anaesthetic consisting of an intravenous dose of propofol sufficient to obtund the eyelid reflex, fentanyl $1 \mu \mathrm{g} / \mathrm{kg}$ and maintenance with isoflurane in a mixture of $66 \%$ nitrous oxide in oxygen. Patients breathed spontaneously via a laryngeal mask airway. Monitoring included automated recording of the arterial pressure, electrocardiogram, pulse oximetry and capnography.

A standard arthroscopy technique was used. Mechanical distraction was applied to the 
Table I. Patient data

\begin{tabular}{|c|c|c|c|}
\hline & Bupivacaine ( $n=17$ ) & Saline ( $n=18$ ) & p-value \\
\hline \multicolumn{4}{|l|}{ Gender } \\
\hline$M: F$ & $8: 9$ & $13: 5$ & 0.18 \\
\hline Age in yrs (range) & $45.9(19$ to 76$)$ & $41.8(20$ to 72$)$ & 0.407 \\
\hline Tourniquet time in min (range) & 44.7 (35 to 65$)$ & 43.6 (30 to 65$)$ & 0.723 \\
\hline Intra-operative fentanyl in $\mu \mathrm{g}$ (range) & $110.3(100$ to 150$)$ & $109.7(100$ to 200$)$ & 0.942 \\
\hline Recovery room analgesia tramadol in mg (range) & 44.1 ( 0 to 250$)$ & $91.7(0$ to 250$)$ & 0.009 \\
\hline $\begin{array}{l}\text { Number of co-codamol tablets taken after discharge } \\
\text { (range) }\end{array}$ & $5.18(0$ to 12$)$ & $6(0$ to 16$)$ & 0.628 \\
\hline
\end{tabular}

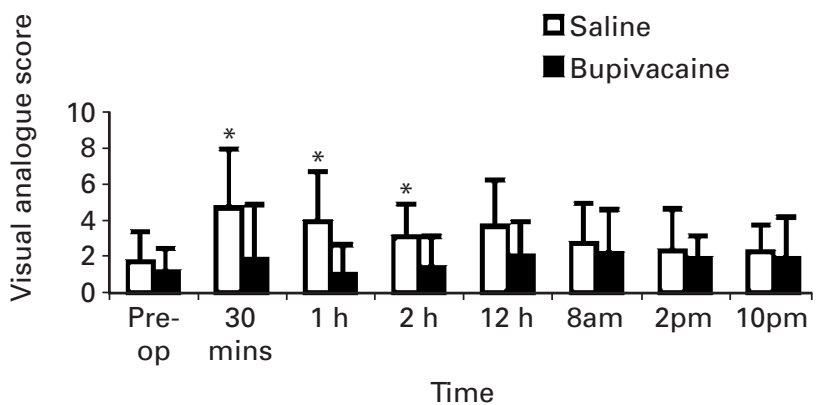

Fig. 1

Visual analogue scale pain scores (mean and SD) as a function of time. Scores measured pre-operatively, 30 minutes, one, two and 12 hours post-operatively on the day of surgery, and at $8 \mathrm{am}, 2 \mathrm{pm}$ and $10 \mathrm{pm}$ on the day after surgery $\left({ }^{*}\right.$ indicates $\left.p<0.05\right)$.

ankle with a Guhl ankle strap soft-tissue distractor (Smith and Nephew, Memphis, Tennessee). The ankle was distended with saline and the anteromedial portal was created with a No. 15 blade and a $2.7 \mathrm{~mm}$ diameter arthroscope inserted. The anterolateral portal was made using a transillumination technique avoiding the superficial peroneal nerve.

At the end of the procedure an intra-articular injection of either $20 \mathrm{ml}$ normal saline or $20 \mathrm{ml} 0.5 \%$ bupivacaine was given as appropriate. The tourniquet was deflated ten minutes later, and this was taken as the start time for the study.

In the recovery room supplementary analgesia of two tablets of co-codamol $30 / 500$ orally, tramadol $50 \mathrm{mg}$ to $100 \mathrm{mg}$ intravenously (IV) up to a maximum of $250 \mathrm{mg}$, and morphine $1 \mathrm{mg}$ to $10 \mathrm{mg}$ IV were available on request. The recovery staff recorded the quantities administered. On discharge, patients were given a ten-day supply of diclofenac (50 mg, eight-hourly, to take regularly), and in addition co-codamol 30/500 (two tablets, six-hourly to take as required). Patients were asked to document the number of co-codamol tablets taken and the time.

The analgesic effect was assessed by a direct subjective measure of pain intensity, using a visual analogue scale (VAS). The requirements for co-codamol as an additional analgesic were also monitored as an indirect indicator of the intensity of the pain. The patients were instructed pre-operatively in the use of the VAS. This was a $100 \mathrm{~mm}$ line with 0 marked as 'no pain' and 100 as 'the worst pain imaginable'. Recordings were made before operation and then on the day ward 30 minutes, 1 hour and 2 hours after release of the tourniquet. The patients then recorded their VAS scores after discharge at 12 hours after operation, and at $8 \mathrm{am}, 2 \mathrm{pm}$ and $10 \mathrm{pm}$ the next day. The operating surgeon (SP) was not involved in recording the VAS or in the collection of the completed forms.

The sample size was estimated by an equation appropriate for comparing two independent group means. The power of the study was calculated prior to commencement of the trial. Based on a standard deviation of $20 \mathrm{~mm}$ for the $\mathrm{VAS}^{7}$ a group of 20 patients would be sufficient to detect a difference of $18 \mathrm{~mm}$ on the VAS at a $5 \%$ level of significance with $80 \%$ power. However, after recruitment of 35 patients, the introduction of new European legislation (EU Directive 2001/20/EC) ${ }^{9}$ necessitated halting the trial to implement fresh documents and procedures. In view of the additional costs and time involved in implementing these practices we did not feel able to continue with the study and the data were unblinded and analysed.

Statistical analysis. The data are shown as mean (SD) values unless otherwise stated, and unpaired two-tailed $t$-tests were used to compare the differences between the groups. Data that were not normally distributed were analysed using the Mann-Whitney U-test. Categorical values were analysed using the chi-squared test. A p value $<0.05$ was considered to be significant. All analyses were carried out using SPSS for Windows version 10 (SPSS Inc., Chicago, Illinois).

\section{Results}

There were no significant differences between the two groups in age, gender or tourniquet time (Table I) and the pre-operative VAS scores were similar for the two groups. Both groups had an equal number of patients who had undergone arthroscopic debridement. The VAS scores were normally distributed. 
The post-operative VAS scores were significantly lower in the bupivacaine group on the day of operation (Fig. 1) 30 minutes $(\mathrm{p}=0.013), 60$ minutes $(\mathrm{p}=0.001)$ and $120 \mathrm{~min}$ utes after the procedure $(\mathrm{p}=0.010)$. There was a trend towards a lower VAS score at 12 hours following arthroscopy $(\mathrm{p}=0.07)$. Although the mean VAS scores were lower for the bupivacaine group on the day after operation, the results were not statistically significant.

The use of IV tramadol for post-operative analgesia in the recovery room was less in the bupivacaine group $(44.1 \mathrm{mg} v \mathrm{~s}$ $91.7 \mathrm{mg}, \mathrm{p}=0.009)$. Four patients $(24 \%)$ in the bupivacaine group required tramadol, as opposed to $15(83 \%)$ in the saline group, and an additional four patients $(22 \%)$ in the saline group also required morphine. The use of cocodamol for additional analgesia following discharge was similar in both groups. One patient in the saline group returned to the Accident and Emergency department for additional analgesia after discharge. There were no sideeffects or complications associated with the use of bupivacaine.

\section{Discussion}

Following arthroscopy of the ankle, pain is routinely managed with oral opioids and anti-inflammatory medication. These are administered by the patient, but may result in inadequate pain relief. Persistent pain may delay discharge from hospital, reduce the ability to participate in a rehabilitation programme and delay recovery. Total or optimal analgesia is difficult to achieve with a single drug or method. A multimodal analgesic regimen utilises the synergistic effects, allowing the use of smaller doses of supplementary analgesia with a concomitant reduction in side effects. ${ }^{10}$

Intra-articular administration of bupivacaine $0.5 \%$ or less does not appear to be detrimental to the articular cartilage and was used because of its extended duration of action compared with that of other local anaesthetics. ${ }^{3,11}$

The study by Rasmussen and Kehlet ${ }^{8}$ assessed the effect of intra-articular glucocorticoid, morphine and bupivacaine and demonstrated an initial analgesic effect, with a prolonged difference between their treatment and placebo groups. This may be accounted for by the addition of morphine and corticosteroid. However, there was one infection in their treatment groups, and in view of this we did not feel that the addition of corticosteroid was prudent.

Bupivacaine is a longer-acting local anaesthetic amide but still has a relatively short half-life of approximately three to four hours. The main benefit of intra-articular bupivacaine was in the first eight hours, and in view of this the addition of other adjuvants, such as an opioid or clonidine, may provide longer-lasting analgesia.

We recommend the use of intra-articular bupivacaine following arthroscopy of the ankle as it significantly reduces the initial levels of pain and the requirements for analgesics after operation. Further studies are required to assess the benefit of other adjuvants.

No benefits in any form have been received or will be received from a commercial party related directly or indirectly to the subject of this article.

\section{References}

1. Ferkel RD, Nuys V, Scranton PE. Arthroscopy of the ankle and foot. J Bone Joint Surg $[$ Am] 1993;75-A:1233-42.

2. Cousins MJ. Prevention of postoperative pain. Procs VIth World Congress on Pain. Elsevier Science Publishers 1991:41-57.

3. Nole R, Munson NM, Fulkerson JP. Bupivacaine and saline effects on articular cartilage. Arthroscopy 1985;1:123-7.

4. Katz JA, Kaeding CS, Hill JR, Henthorn TK. The pharmokinetics of bupivacaine when injected intra-articularly after knee arthroscopy. Anesth Analg 1988;67:872-5.

5. Chirwa SS, MacLeod BA, Day B. Intraarticular bupivacaine (Marcaine) after arthroscopic meniscectomy: a randomised double-blind controlled study. Arthroscopy 1989:5:33-5.

6. Raja SN, Dickstein RE, Johnson CA. Comparison of postoperative analgesic effects of intraarticular bupivacaine and morphine following arthroscopic knee surgery. Anesthesiology 1992;77:1143-7.

7. Laurent SC, Nolan JP, Pozo JL, Jones CJ. Addition of morphine to intra-articular bupivacaine does not improve analgesia after day-case arthroscopy. $B \mathrm{~J}$ Anaesth 1997;78:163-8.

8. Rasmussen S, Kehlet H. Intraarticular glucocorticoid, morphine and bupivacaine reduces pain and convalescence after arthroscopic ankle surgery: a randomized study of 36 patients. Acta Orthop Scand 2000;71:301-4.

9. No authors listed. Directive 2001/20/EC of the European parliament and of the council of 4 April 2001. Official Journal of the European Communities L121/37-44.

10. Kehlet H, Dahl JB. The value of "multimodal" or "balanced analgesia" in postoperative pain treatment. Anesth Analg 1993;77:1048-56.

11. Moore DC, Bridenbaugh LD, Thompson GE, Balfour RI, Horton WG. Bupivacaine: a review of 11,080 cases. Anesth Analg 1978;57:42-53. 